\title{
INFLUENCE OF THE ELECTRONIC SHELL STRUCTURE ON THE ELASTIC SCATTERING OF HEAVY IONS ${ }^{\text {is }}$
}

\author{
H. HARTUNG, B. FRICKE, H. LENZ and W.-D. SEPP \\ Department of Physics, University of Kassel, D-3500 Kassel, Fed. Rep. Germany
}

Received 14 June 1982

\begin{abstract}
The interatomic potential of the system I-I at intermediate and small distances is calculated from atomic DFS electron densities within a statistical model. Structures in the potential, due to the electronic shells, are investigated. Calculations of the elastic differential scattering cross section for small angles and several keV impact energies show a detailed peak pattern which can be correlated to individual electronic shell interaction.
\end{abstract}

The potential energy surface for elastic scattering between atomic particles, known as the interatomic potential, has been subject to investigation for many years [1-4]. The main features are well described by analy tic forms like the Bohr potential [5] or, as a better approximation, by the Lenz-Jensen potential [6]. The numerical Thomas-Fermi approach has been applied to diatomic systems by Firsov [7]. All these models are applicable quite well to heavy ion scattering experiments, where the impact energy is above the $\mathrm{keV}$ range, and scattering angles are not too small. However, at $\mathrm{cm}$ angles in the forward scattering region, with energies of about $100 \mathrm{keV}$, the inflection point of the classical trajectory is reached at an internuclear distance where the inner atomic shells of the scattering partners overlap. Here the electronic shell structure of the scattering system has to be taken into consideration. Experiments under these scattering conditions by Loftager et al. [4] have shown that the differential cross section for elastic collisions has a detailed structure. This can be understood as an effect due to the spatial electron distribution in the two-centre many-electron system.

As a first step we have calculated the interatomic potential of the system $\mathrm{I}-\mathrm{I}$ as an example in a den-

\footnotetext{
is Supported by Gesellschaft für Schwerionenforschung, Darmstadt.
}

sity functional àpproach. The total system energy in this model is

$E=V_{\mathrm{NN}}+V_{\mathrm{Ne}}+V_{\mathrm{ee}}+T_{\mathrm{e}}$,

where

$V_{\mathrm{NN}}=Z_{1} Z_{2} / R$

is the potential energy of the bare nuclei,

$V_{\mathrm{Ne}}=-\int\left(\frac{Z_{1}}{\left|r-r_{1}\right|}+\frac{Z_{2}}{\left|r-r_{2}\right|}\right) \rho(r) \mathrm{d}^{3} r$

the electron-nucleus interaction energy,

$V_{\mathrm{ee}}=\frac{1}{2} \int \frac{\rho(r) \rho\left(r^{\prime}\right)}{\left|r-r^{\prime}\right|} \mathrm{d}^{3} r \mathrm{~d}^{3} r^{\prime}-\kappa_{\mathrm{a}} \int \rho^{4 / 3} \mathrm{~d}^{3} r$

the electron-electron interaction energy, consisting of the direct part and the exchange part in the Slater approximation, and

$T_{\mathrm{c}}=\kappa_{\mathrm{k}} \int \rho^{5 / 3} \mathrm{~d}^{3} r$

the kinetic energy term. This last term is based on the statistical TF theory of the atom [8]. The constants $\kappa_{\mathrm{a}}$ and $\kappa_{\mathrm{k}}$ are in atomic units

$\kappa_{\mathrm{a}}=\alpha_{\mathrm{x}} \frac{3}{2}(3 / \pi)^{1 / 3}, \quad \kappa_{\mathrm{k}}=\frac{3}{10}\left(3 \pi^{2}\right)^{2 / 3}$,

where we use $\alpha_{\mathrm{x}}=0.7$.

For the electron density $\rho$ we choose the sum of 
atomic densities $\rho=\rho_{1}^{\mathrm{DFS}}+\rho_{2}^{\mathrm{DFS}}$, where the atomic densities have been calculated by using good SCF DFS wavefunctions. To obtain the scattering potential energy we have to subtract from the total system energy the total energies of the separated atoms $V$ $=E-E_{1}-E_{2}$. The potential energy curve $V(R)$ results from calculating $E$ at the internuclear separation $R$.

Since the potential structure due to the electronic shell structure in an absolute plot is very small, we choose a relative plot where only the difference $\left(V-V_{\mathrm{LJ}}\right) R$ to the Lenz-Jensen [6] potential $V_{\mathrm{LJ}}$ is drawn as function of $R$. This function is shown in fig. 1 . Here the electronic shell structure in the region between 0.1 and 2.0 au can be seen quite well. The minimum at 0.02 au is due to the analytic form of the Lenz-Jensen potential. To compare these results with experiments we have calculated the elastic scattering function $\theta(b)$ in the centre-of-mass system, classically:

$$
\begin{aligned}
& \theta(b)=\pi \\
& -2 b \int_{R_{0}}^{\infty} r^{-2}\left[1-b^{2} / r^{2}-V(r) / E_{0 \mathrm{~cm}}\right]^{-1 / 2} \mathrm{~d} r,
\end{aligned}
$$

where $b$ is the impact parameter, $R_{0}$ the distance of closest approach, and $E_{0 \mathrm{~cm}}$ the c.m. impact energy. From the scattering function we get the elastic differential scattering cross section

$\mathrm{d} \sigma / \mathrm{d} \Omega=(b / \sin \theta)|\mathrm{d} \theta / \mathrm{d} b|^{-1}$.

We have calculated cross sections for c.m. impact energies from 25 to $400 \mathrm{keV}$. In fig. 2 the results are shown in a scaled plot where the small angle cross sections for different impact energies fall on one curve. This scaling law results from the LNS-theory

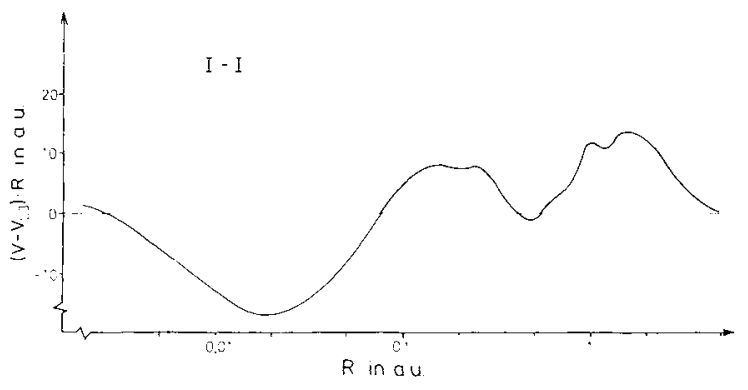

Fig. 1. Relative scattering potential for the system I-I.

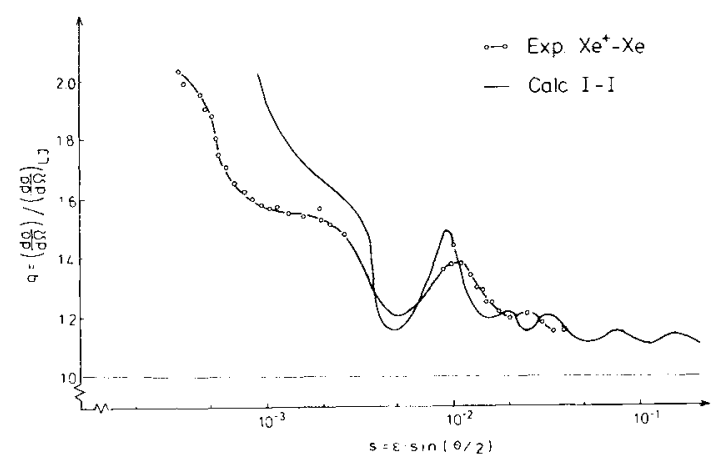

Fig. 2. Differential scattering cross sections for $\mathrm{Xe}^{+}-\mathrm{Xe}$ (experiment, ref. [4]) and I-I (theory, this work).

of Lindhard et al. [2]. The abscissa is the dimensionless quantity $s=\epsilon \sin (\theta / 2)$, where $\theta$ is the c.m. scattering angle and $\epsilon$ a reduced energy $\epsilon=\left(E_{0 \mathrm{~cm}} a\right) /$ $\left(Z_{1} Z_{2}\right)$. The screening length $a$ is defined by $a$ $=0.8853\left(Z_{1}^{2 / 3}+Z_{2}^{2 / 3}\right)^{-1 / 2}$. The ordinate is the reduced differential scattering cross section $q=(\mathrm{d} \sigma /$ $\mathrm{d} \Omega) /(\mathrm{d} \sigma / \mathrm{d} \Omega)_{\mathrm{LJ}}$, where $L J$ indicates the value for the Lenz--Jensen potential. The calculated scattering cross sections give a structure that is comparable to the one measured [4] in the system $\mathrm{Xe}^{+}-\mathrm{Xe}$.

The relatively good agreement between experiment and theory in fig. 2 shows that for the first time the general behaviour of elastic heavy ion collisions can be described theoretically. It is even possible to correlate the potential structures in fig. 1 with the peaks in fig. 2. Especially the structures from the inner shells, e.g. $\mathrm{M}-\mathrm{N}$ shell interaction for $s \approx 10^{-2}$ as well as the $\mathrm{L}-\mathrm{N}$ and $\mathrm{M}-\mathrm{M}$ interactions for larger $s$, can be correlated to experimental data in their absolute values. It would be interesting to have measurements for the $\mathrm{L}-\mathrm{L}$ and even $\mathrm{K}-\mathrm{L}$ interaction as well, which lead to the theoretical peaks at $s \approx 10^{-1}$. We interpret the deviations of the peak positions from the measured values as quasimolecular effects which are not included in our model. To test this the same calculation has to be done with an ab initio self-consistent field internuclear potential for the two-centre many-electron system. Here the rearrangement of the atomic orbitals during the collision process is taken into account. Such calculations are in preparation. 


\section{References}

[1] For a general review see I.M. Torrens, Interatomic potentials (Academic Press, New York, 1972).

[2] J. Lindhard, V. Nielsen and M. Scharff, K. Dan. Vid. Selsk. Mat. Fys. Medd. 36 (1968) No. 10.

[3] V.K. Nikulin, Zh. Tekh. Fiz. 41 (1971) 41 [Sov. Phys. Tech. Phys. 16 (1971) 281.

[4] P. Loftager, F. Besenbacher, O.S. Jensen and V.S. S $\phi$ rensen, Phys. Rev. A20 (1978) 1443.
[5] N. Bohr, K. Dan. Vid. Selsk. Mat. Fys. Medd. 18 (1948) No. 8.

[6] W. Lenz, Z. Phys. 77 (1932) 713 ; H. Jensen, Z. Phys. 77 (1932) 722.

[7] O.B. Firsov, Zh. Eksp. Teor. Fiz. 33 (1957) 696 [Sov. Phys. JETP 6 (1958) 534].

[8] P. Gombas, Handbuch der Physik, Vol. 36, ed. S. Flügge (Springer, Berlin, 1956) p. 109. 